\title{
Intégration socioprofessionnelle et mobilité internationale. Le cas des ingénieurs français ayant emprunté l'ARM France-Québec
}

«Immigrant incorporation encompasses a broader range of policies and personal experiences of social belonging in a receiving country. » van Riemsdijk, Basford et Burnham (2016: 22)

\section{Introduction}

Cet article s'inscrit dans une démarche de recherches en cours depuis 2012, dont les objectifs sont de comprendre et analyser l'évolution du système professionnel québécois dans sa gestion de l'admission de professionnels formés à l'étranger. Plus précisément, nous retenons ici le cas des professionnels français admissibles aux Arrangements de reconnaissance mutuelle (ARM) France-Québec pour documenter leur parcours d'intégration. En effet, nous examinons les parcours d'ingénieurs français, en comparant les discours d'acteurs du système professionnel ${ }^{1}$ et ceux d'ingénieurs français ayant eu recours à l'Arrangement de reconnaissance mutuelle (ARM) France-Québec. Pour ce faire, nous allons d'abord situer cet accord dans le paradigme de la mobilité (Pellerin, 2011; Papademetriou et al., 2009). Ce modèle de gestion de la migration des travailleurs facilite a priori l'intégration au marché du travail des professionnels formés en France. Nous verrons toutefois que plusieurs obstacles apparaissent sur le chemin de l'intégration, qui est d'abord conçue en termes économiques, laissant de côté la dimension sociale et familiale de ces immigrants. Plusieurs recherches tendent à appuyer

\footnotetext{
${ }^{1}$ Ces acteurs du système professionnel sont associés à des organisations publiques (par exemple, l'Office des professions du Québec), para-publiques (ordres professionnels) ou privées (associations de professionnels, groupes d'entraide de professionnels formés à l'étranger, etc.).
} 
ce point de vue, mais soulignent du même souffle, le manque de connaissances à cet égard (Chicha, 2010; van Riemsdijk, Basford et Burnham, 2016; Friesen, 2011). Cette problématique devient d'autant plus saillante dans le contexte d'émergence des Arrangements de reconnaissance mutuelle (ARM) comme modèle de mobilité des professionnels formés à l'étranger.

Après avoir précisé notre cadre conceptuel et méthodologique, nous décrirons les données issues de deux études de cas cumulant les propos et points de vue d'ingénieurs français et d'acteurs du système professionnel. L'analyse sera l'occasion de jeter un regard critique sur l'action de ces acteurs en faisant ressortir les principales dimensions de l'intégration socioprofessionnelle de ces immigrants et l'impact de certaines incohérences systémiques face à l'objectif initial d'intégration optimale des professionnels formés à l'étranger.

\section{Les Arrangements de reconnaissance mutuelle (ARM) : un accord pour la mobilité internationale de professions réglementées}

L'Entente en matière de reconnaissance mutuelle des qualifications professionnelles France-Québec s'inscrit dans les nouvelles tendances internationales d'organisation du travail et de gestion des flux migratoires. Ces tendances évoquent l'apparition d'un nouveau paradigme de la migration, celui de la mobilité (Papademetriou, Sumption et Somerville, 2009 cités par Pellerin, 2011). Dans ce sens, « la quête d'un emploi sera au cœur de la plupart des mouvements migratoires du XXIe siècle » (OIM, 2008).

Le paradigme de la mobilité vise à comprendre la migration dans un contexte socioéconomique plus large (Noiriel, 2001, 2008; Larbiou, 2003, 2008), qui semble s'imposer 
depuis les années 2000. Ce paradigme renvoie à un modèle économique, social et politique néolibéral, qui se traduit par la « libéralisation des flux de capitaux, des biens et des services, en touchant davantage les pays industrialisés et certaines catégories de migrants » (Pellerin, 2011). Les États et les organismes internationaux, régionaux et nationaux, s'inscrivent dans ce paradigme de la mobilité, par leurs lois, leurs politiques et leurs pratiques en matière d'immigration. Ainsi, de nouvelles formes de gouvernance, comme les Arrangements de reconnaissance mutuelle (ARM), apparaissent dans la gestion du travail et de l'immigration à l'échelle globale (Cohen, 2006).

\section{Les Arrangements de reconnaissance mutuelle (ARM): Genèse et principes directeurs}

L'entente-cadre pour les ARM France-Québec a été signée en 2008. Elle vise à «pallier aux pénuries de main-d'oeuvre qualifiée et à répondre plus efficacement aux besoins des entreprises québécoises et françaises » (MRIF, 2011), prévoyant une reconnaissance mutuelle pour 64 professions au Québec, dont 28 sont régies par des ordres professionnels. Suite à cette signature, les acteurs des systèmes professionnels français et québécois ont été interpellés pour examiner rapidement la faisabilité d'une telle entente mutuelle.

Notons que pour rendre les ARM négociés effectifs au Québec, ils devaient être traduits en réglementation spécifique et appliqués pour chacune des professions, en cohérence avec les lois du système professionnel québécois. Le modèle emprunté par les ARM se différencie de l'approche traditionnelle d'admission aux ordres professionnels par des travailleurs formés à l'étranger. En effet, au lieu de traiter ces demandes une à la fois, une analyse comparative de l'exercice de la profession dans chacune des juridictions a lieu. 
Cette analyse à visée générique définit, pour tout candidat éligible, les stages et/ou formations complémentaires à suivre pour terminer l'entrée en profession dans la société d'accueil.

Chacun des ARM comporte cinq grands principes directeurs : la protection du public; le maintien de la qualité du service professionnel; l'équité, la transparence et la réciprocité; l'effectivité des qualifications professionnelles mutuellement reconnues; et le respect des normes associées à l'usage de la langue française (via l'Office québécois de la langue française, OQLF). Parmi ces cinq principes, la protection du public est au cœur de l'ARM et du mandat des ordres professionnels. Les processus mis en place par les ARM constituent une manière accélérée d'obtenir un permis d'exercice au Québec, par rapport aux processus empruntés par d'autres professionnels formés à l'étranger, tout en visant le maintien de la protection du public.

Il existe, théoriquement, une tension entre le principe de protection du public, tel que promulgué par les divers ordres professionnels, et le principe d'effectivité de reconnaissance des compétences professionnelles (MRIF, 2011). L'objectif poursuivi par les règlements des ARM est de trouver les mécanismes favorisant l'accès des professionnels formés à l'étranger au système professionnel québécois tout en maintenant la protection du public, par des mesures de compensation ${ }^{2}$. À cet effet, « pour combler les différences substantielles en ce qui a trait aux titres de formation ou aux programmes d'apprentissage, l'Entente Québec-France établit que les ordres doivent déterminer si ces

\footnotetext{
2 «L'Entente entre la France et le Québec en matière de reconnaissance mutuelle des qualifications professionnelles prévoit qu'un ordre professionnel procède à l'examen comparatif des qualifications professionnelles. Cet examen vise à déterminer, le cas échéant, les conditions de reconnaissance de ces qualifications qui seront fixées dans un Arrangement de reconnaissance mutuelle (ARM) pris en vertu de cette entente et dans un règlement de mise en œuvre pris en vertu de l'article 93 c.2) du Code des professions » (CIQ, CREPUQ, $2010: 1$ ).
} 
différences peuvent être compensées par l'expérience professionnelle du demandeur. Si les ordres jugent que cette mesure est inadéquate, ils peuvent envisager d'autres mesures de compensation, tels le stage d'adaptation, l'épreuve d'aptitude ou la formation complémentaire » (Houle, $2015: 3)$.

Ces mesures peuvent, dans certains cas, poser question quant à leur raison d'être et à leur pertinence de la part des professionnels étrangers. Le point de divergence se trouverait dans l'ambiguïté de la notion de «différences substantielles » ainsi que dans le fait que ce jugement reste à la discrétion des ordres. C'est l'approche dite de reconnaissance «permis sur permis ». Les exigences sont cependant modulées, de diverses façons, pour correspondre aux caractéristiques de la formation et de l'exercice de la profession, en France et au Québec.

Quant aux ingénieurs, une liste des établissements reconnus de formation d'ingénieurs a été établie et est mise à jour de façon périodique. Une fois l'éligibilité établie, chaque candidat diplômé de France doit compléter une année sous la supervision d'un ingénieur membre de l'Ordre des Ingénieurs du Québec (OIQ). Au terme de cette année et après la réussite d'un examen obligatoire ${ }^{3}$, l'ingénieur est admis au tableau de l'OIQ ${ }^{4}$.

\footnotetext{
${ }^{3}$ «L'examen professionnel n'est pas de nature technique. Il a plutôt pour but de vérifier les connaissances sur :

- le droit professionnel québécois;

- les principes de la pratique professionnelle, les notions d'éthique et de professionnalisme, ainsi que les autres obligations professionnelles;

- les aspects juridiques de base jugés essentiels à l'exercice de la profession. »

Les modalités de l'examen, les conditions de réussite et les recours en cas d'échec sont ensuite précisées (OIQ, 2017a).

${ }^{4}$ « Dès la réception de votre demande de permis, le Service d'accès à la profession s'assure que le domaine inscrit sur votre diplôme d'ingénieur est identique en tout point à celle [sic] qui est indiqué [sic] sur la liste des programmes français agréés par l'Ordre. De plus, il s'assure que tous les documents reçus sont conformes à ceux qui sont exigés et vous avise si des documents supplémentaires doivent être fournis.

Le Service d'accès à la profession détermine si vous êtes admissible en vertu de l'ARM ou si vous devez obtenir une équivalence de formation.
} 


\section{La démarche méthodologique : L'étude de cas}

Nous examinons l'intégration socioprofessionnelle des ingénieurs diplômés en France ayant eu recours à l'Arrangement de reconnaissance mutuelle (ARM) France-Québec depuis son entrée en vigueur le 18 juillet 2013. Plus précisément, nous analysons les facilitateurs et les obstacles à l'intégration socioprofessionnelle et leurs effets sur la rétention de ces travailleurs et leurs familles. Au passage, ceci nous amène à nous pencher sur le rôle et les dynamiques entre les différents acteurs impliqués (gouvernements et institutions publiques, ordres professionnels, entreprises, société civile, etc.) et l'efficacité des politiques régulant le marché de l'emploi et l'immigration en facilitant l'entrée en pratique ainsi que l'intégration dans la société d'accueil. Nous avons d'abord mené des entrevues avec des informateurs-clés; en parallèle, nous nous sommes documentés sur différents aspects de la profession d'ingénieur en France et au Québec. Les entrevues avec les ingénieurs ont été effectuées à l'été 2014, avec la collaboration de l'Ordre des ingénieurs du Québec. Bien que des rencontres avec des membres de l'entourage professionnel avaient été prévues au devis de recherche, seul un supérieur hiérarchique commun à deux de ces ingénieurs a accepté de participer à une entrevue. Cette difficulté, ainsi que la mise en vigueur récente du règlement concernant les ingénieurs pendant la recherche, ont limité nos possibilités de recrutement. Les

Si vous êtes titulaire d'un diplôme visé par l'ARM et que votre dossier est complet, il faudra prévoir un délai de traitement d'environ 6 à 8 semaines afin que l'Ordre vous délivre un permis restrictif temporaire en génie (DPRTG : détenteur d'un permis restrictif temporaire en génie).

Par la suite, vous devrez démontrer :

- la preuve de trois années d'expérience pertinente, dont au moins une effectuée au Canada sous la direction et la surveillance d'un ingénieur ET

- réussir l'examen professionnel. Le détenteur d'un DPRTG doit exercer sous la direction et la surveillance immédiates d'un ingénieur, c'est-à-dire une personne qui détient un permis d'ingénieur et qui est inscrite au tableau de l'Ordre. » (OIQ, 2017b). 
entrevues étaient de type semi-dirigé, d'une durée moyenne d'une heure et demie, orientées par une grille d'entrevue et validées au préalable par le Comité d'éthique à la recherche de l'INRS-Centre $\mathrm{UCS}^{5}$ et de l'Université de Montréal.

Les données concernant les ingénieurs sont issues des 14 entrevues suivantes :

- Huit entrevues auprès d'ingénieurs diplômés en France et ayant eu recours à l'ARM (depuis juillet 2013) (trois ingénieurs civils, trois ingénieurs électriques et deux ingénieurs d'autres domaines du génie),

- Six entrevues auprès d'acteurs du système professionnel, soit : trois entrevues auprès d'acteurs du système professionnel québécois, deux entrevues auprès d'associations et d'organismes collaborant à l'intégration de travailleurs immigrants qualifiés, notamment des ingénieurs français, et une entrevue auprès d'un représentant de l'employeur de deux des ingénieurs rencontrés.

Des huit ingénieurs français, tous étaient d'immigration récente (moins de 5 ans), cinq se trouvaient à Montréal et trois dans d'autres régions du Québec - dont la seule femme rencontrée. Plus de la moitié (5/8) étaient en début de carrière, après quelques années de pratique en France, tandis que trois d'entre eux sont arrivés au Québec en milieu de carrière.

\section{L’intégration socioprofessionnelle des ingénieurs diplômés en France}

Il semble y avoir, depuis un certain nombre d'années, un intérêt grandissant au chapitre de la mobilité internationale des travailleurs qualifiés dans les différentes régions du monde (Weygold, Berset et al., 2001). L'une des principales raisons de cet intérêt grandissant serait associée au besoin de saisir davantage l'impact de la présence des

\footnotetext{
${ }^{5}$ Institut national de recherche scientifique, Centre Urbanisation Culture Société.
} 
professionnels formés à l'étranger dans le développement économique et social des sociétés d'accueil. À cet effet, Weygold, Berset et al. (2001: 117) soulèvent que «the best qualified immigrants support the economic development of the country and are thought to have the strongest capacity for social integration ».

Toutefois, tel que la littérature plus récente le soulève, il existe encore une pénurie d'études portant sur l'intégration socioculturelle des migrants qualifiés (van Riesmdijk, Basford et Burnham, 2016). L'une des raisons de cette pénurie semble associée à l'idée que cette catégorie d'immigrants serait autonome dans son intégration à la société d'accueil (Alba et Nee, 1997; Portes et Böröcz, 1989). Cette lacune est encore plus évidente à l'égard des travailleurs migrants touchés par une entente de reconnaissance mutuelle, par exemple l'Arrangement de reconnaissance mutuelle (ARM) France-Québec. Il existe en effet très peu d'écrits touchant les ARM en général (Bédard, 2014). Les principaux documents portent sur de grands ensembles et demeurent essentiellement descriptifs, tels que les rapports récents du Migration Policy Institute ${ }^{6}$ (Mendoza et al., 2017). On trouve encore moins de documents sur l'expérience de professionnels ayant eu recours aux ARM France-Québec, du fait de la nouveauté des règlements consacrant leur mise en vigueur ${ }^{7}$.

La mobilité des travailleurs hautement qualifiés

\footnotetext{
${ }^{6}$ Le Migration Policy Institute (MPI ) crée en 2001 à Washington, DC se dédie à l'analyse de la mobilité internationale. Il élabore des analyses ainsi que des évaluations de politiques migratoires et de réfugiés aux niveaux local, national et international. Il vise à répondre de manière pragmatique aux défis et opportunités des flux migratoires. Voir: https://www.migrationpolicy.org/.

${ }^{7}$ On compte quelques articles présentant les particularités de certains ARM (Mercier, 2012; Dumas-Martin, 2014). Dans une analyse politique des ARM, Dumas-Martin (2014) souligne entre autres qu'il s'agit d'une entente et non d'un accord, ce qui, dans le langage politique, sous-entend un arrangement plus informel qu'un accord ne le serait. Son bon fonctionnement est davantage le résultat d'un leadership politique. De même, les éventuels manquements ou écarts seraient sanctionnés à un niveau plutôt politique que juridique.
} 
De plus, avec le «paradigme de la mobilité », la circulation des travailleurs, sujette aux besoins du marché économique international et de la production, devient « un moyen servant une fin, celle de la distribution optimale dans l'économie» (Pellerin, 2011 : 63). Ainsi, l'accent mis sur l'aspect économique des migrations des professionnels formés à l'étranger contribue à la non-prise en compte d'autres dimensions essentielles à l'intégration de ces immigrants. Dans cet espace mobile, les individus sont perçus comme des « éléments individuels post-sociaux, atomisés et en apesanteur, mus par des forces du marché mondial » (Meyer, Kaplan et Charum, 2001 : 342). La dimension socioculturelle - individuelle et familiale ${ }^{8}$ - n'est pas prise en compte par cette logique de la mobilité dans laquelle les Arrangements de reconnaissance mutuelle (ARM) s'insèrent. Cependant, plusieurs études au Canada et ailleurs soulèvent l'importance d'examiner les dimensions familiales et sociales de l'immigration (Nedelcu, 2005; Favell, Feldblum et Smith, 2007; Friesen, 2011).

La notion de mobilité selon Pellerin (2011) englobe l'expérience générale de l'homo economicus, dans la mesure où elle devient la stratégie universelle de promotion sociale en plaçant l'individu au coeur des responsabilités consacrant son intégration sociale, notamment à travers le développement des réseaux sociaux. Cette vision économiste, inspirée de la théorie économique orthodoxe et de théories utilitaristes du choix rationnel (Rawls, 1971), qui considère les individus comme des êtres rationnels mus par leurs seuls intérêts personnels, présente des lacunes importantes quant à la compréhension du parcours socioprofessionnel des immigrants qualifiés. L'économie n'est pas indépendante des sphères juridiques, politiques et culturelles des individus. Ces sphères concourent à

\footnotetext{
${ }^{8}$ Notons que la plupart des ingénieurs rencontrés sont au Québec avec leurs conjoints/es et leurs enfants et que le choix de s'installer au Québec répond à un projet professionnel, mais aussi familial.
} 
structurer les différents schèmes d'action des acteurs par les milieux sociaux fréquentés (famille, école, groupes de pairs, institutions culturelles, médias, etc.) (Bourdieu, 2000; Lahire, 2012).

Une vaste littérature portant sur l'intégration des travailleurs qualifiés, notamment en Europe, porte sur les stages à court terme des travailleurs expatriés (Beaverstock, 2011; Findlay et al. 1996; Walsh, 2006), les transferts intracompagnies (Beaverstock, 2005; Millar et Salt, 2008), et les travailleurs d'affaires (Faulconbridge et al. 2009). Ces études mettent l'emphase sur la nature temporaire et internationale de l'immigration. D'autres tentent de comprendre les politiques étatiques d'attraction des migrants qualifiés (Kofman, 2005; Hawthorne 2013, 2015). Ces politiques se basent sur le recrutement des travailleurs qualifiés, mais n'examinent pas le parcours à long terme et la rétention dans le pays d'accueil. En dépit d'un certain privilège quant à leur statut, les travailleurs qualifiés doivent s'ajuster aux normes et aux pratiques liées à la profession du pays d'accueil (Walsh, 2006).

Quant aux ingénieurs diplômés à l'étranger, les études les plus récentes soulèvent que ceux-ci rencontrent plus de difficultés à décrocher un poste par rapport à ceux qui ont obtenu leur diplôme au Québec. Par exemple, le taux de chômage des professionnels formés à l'étranger membres de l'Ordre était de $6,5 \%$, comparativement à 2,5\% pour l'ensemble des membres en 2013. Les difficultés sont surtout le lot des ingénieurs juniors issus de l'étranger, dont l'entrée s'avère plus difficile que les jeunes formés au Québec. Cependant, une fois le processus d'admission à l'OIQ complété, le taux d'emploi en génie tend à être équivalent à celui des ingénieurs formés au Québec. Aussi, on note que 
certains professionnels formés à l'étranger ont même un avantage du fait de leur expérience antérieure?

Près des deux tiers $(64,8 \%)$ de l'ensemble des professionnels en génie occupent un emploi qui nécessite d'être membre de l'OIQ. Néanmoins, selon cette même étude, le fait d’être membre de l'OIQ ne se traduirait pas forcément en termes de gain salarial :

«Il n’y a pas de différence significative entre les résultats de salaires et de rémunération globale selon ce critère [être membre de 1’Ordre], sauf pour les 10\% les mieux rémunérés; c'est un avantage salarial de 6,26\% » (Demers, $2015: 12$ ).

Autant aux États-Unis qu'au Canada et au Québec, plusieurs recherches soulèvent l'importance des réseaux interpersonnels et informels dans la qualité de l'insertion professionnelle (Arcand, Lenoir-Achdjian et Helly, 2009; Gauthier, 2013; Lin, 1999; Pellerin, 2011). Dans une étude portant sur l'intégration en emploi d'ingénieurs diplômés à l'étranger au Canada, Friesen (2011) a documenté l'importance de l'apprentissage par des ingénieurs formés à l'étranger des codes de comportement propres au milieu de travail local. Cette étude montre notamment l'écart variable selon le pays d'origine dans la culture du travail, les rapports hiérarchiques, la place ou l'absence de la sphère privée dans les interactions professionnelles, etc. À cet égard, l'apprentissage s'avère nécessaire pour interpréter adéquatement les dynamiques et rapports hiérarchiques ou sociaux en milieu de travail au pays d'accueil.

Comprendre l'intégration comme processus multidimensionnel

Considérant ces pistes de questionnement et à partir d'un cadre théorique et conceptuel issu de la théorie sociologique des systèmes sociaux (Lockwood, 1964), nous avons

\footnotetext{
${ }^{9}$ http://asso-ing.ca/le-chomage-chez-les-ingenieurs-atteint-un-sommet-historique/
} 
développé un modèle analytique nous permettant de manière empirique de répondre aux objectifs ce cette étude.

Nous partons d'une compréhension de l'intégration comme processus et état à appréhender par rapport aux multiples dimensions ou sphères faisant partie d'une structure sociale plus large. Ce processus comprend également une composante interactive entre les différents systèmes ou dimensions ainsi qu'entre les acteurs et les institutions qui les composent. Ainsi, l'intégration des individus à une structure sociale déterminée est appréhendée ici sous une approche multiniveau et multisectorielle.

L'approche systématique de Lockwood (1964) nous permet, de façon empirique, de mener une analyse du «système d'intégration $»^{10}$ comme notion processuelle et multidimensionnelle.

Plusieurs modélisations ont été proposées afin d'appréhender de manière empirique l'intégration des individus, notamment des populations immigrantes au sein d'une structure sociale (Esser, 2004 dans Heckmann, 2005). Deux aspects caractérisent essentiellement la plupart de ces modèles: la conception multidimensionnelle de l'intégration (Esser, 2004 dans Heckmann, 2005; Heckmann et Schnapper, 2003; Heckmann, 2005) et l'accent porté sur les conséquences de l'intégration de ces immigrants dans la structure sociale de la société d'accueil (Gordon, 1978; Myrdal, 1944; Rex et Tomlinson, 1979).

\footnotetext{
10 "System integration is that form of integration in a system that works relatively independent of the motives, goals and relations of individual actors, quite often against their motives and interests. System integration is integration of social systems via institutions and organisations, via the state, the legal system, markets, corporate actors or money. It is a mostly anonymous form of integration. Social integration stands for the inclusion of new individual actors in a system, for the creation of mutual relationships among actors and for their attitudes to the social system as a whole" (Lockwood, 1964 dans Heckmann, 2005: 9).
} 
À partir de cette littérature et de manière spécifique, inspirés des travaux de Heckmann (2005), nous avons donc développé un modèle analytique de l'intégration des ingénieurs diplômés en France au marché de l'emploi et à la société québécoise.

Heckmann (2005) identifie l'existence de cinq dimensions explicatives de l'intégration sociale des immigrants, soit : 1) une dimension structurelle, 2) une dimension culturelle, 3) une dimension interactive ou relationnelle, 4) une dimension identitaire et 5) une dimension qui conçoit l'intégration comme un processus d'apprentissage et de socialisation.

Pour cet article, nous avons décidé de regrouper ces cinq dimensions en deux grandes catégories. La première comprend tous les aspects structurels de l'intégration (dimension structurelle) et la seconde, l'ensemble des aspects socioculturels et relationnels, ainsi que ceux reliés à la socialisation dans la société d'accueil (dimension culturelle et relationnelle).

Nous définissons la dimension structurelle comme l'ensemble des lois, règlements, procédures administratives et la gouvernance concernant l'immigration, le marché de l'emploi, l'admission à la profession ainsi que l'accès aux institutions de la société d’accueil. La dimension culturelle fait référence à l'apprentissage et la compréhension des codes culturels, liés à l'emploi autant qu'à la façon de faire de la société d'accueil en général. La dimension relationnelle comprend l'ensemble des relations personnelles et professionnelles des migrants avec les membres de la société d'accueil (liens sociaux avec les collègues, mais aussi avec leurs supérieurs, liens d'amitié, familiaux, etc. ainsi que leur implication dans la société civile). La raison d'opérationnaliser ainsi ces 
dimensions répond à une compréhension intégrée des processus de socialisation, qui relèvent à la fois des aspects structurels, culturels, que relationnels et identitaires.

\section{Le processus d'intégration socioprofessionnelle}

\section{a) la dimension structurelle}

Cette dimension comprend l'ensemble des structures gouvernementales et des systèmes professionnels, les mécanismes de gouvernance, les dynamiques entre les acteurs et les balises normatives et juridiques ayant un lien avec les processus d'intégration socioprofessionnelle des ingénieurs éligibles à l'Arrangement de reconnaissance mutuelle (ARM) France-Québec.

Concrètement, nous analysons : 1) l'effet de certaines politiques d'immigration, d'accueil et d'intégration des travailleurs qualifiés au Québec, 2) la démarche propre à l'ARM des ingénieurs, 3) l'adéquation entre le discours politique et les réalités sur le terrain (autant quant au marché de l'emploi qu'au niveau social), 4) l'exercice de la profession et ses différences entre le Québec et la France et 5) l'effet d'autres politiques dans l'intégration socioprofessionnelle des travailleurs - notamment l'éducation et la reconnaissance des diplômes, l'accès aux services sociaux, au logement, etc.

\section{Analyse}

Il existe une panoplie d'acteurs et d'institutions de nature diverse ayant un rôle dans l'élaboration et la mise en place de l'ARM ainsi que de l'ensemble des démarches en lien avec l'établissement et l'intégration socioprofessionnelle au Québec des ingénieurs diplômés en France en vertu de cette entente. Parmi ces acteurs, nous avons constaté que les employeurs et les services mis en place par ces derniers pour l'accueil et l'intégration des professionnels français jouent un rôle très important dans leur processus d'intégration 
socioprofessionnelle. Nous avons pu constater par notre démarche de terrain l'existence d'entreprises ayant développé une expertise dans l'accueil d'ingénieurs français. Ainsi, dans ces cas, une personne-ressource dans l'entreprise reçoit le mandat d'accompagner et soutenir l'intégration des employés français autant dans le milieu de travail que dans la société québécoise, incluant les démarches concernant la recherche de logement, d'un médecin, etc.

"On n'a pas, nous, chez nous, de programme d'accueil en tant que tel. On y va à la pièce. Moi je prends la peine de m'assurer, bien, comment il arrive, où il arrive, est-ce qu'il y a quelqu'un qui l'accompagne à son arrivée. Je l'ai fait une fois, un individu est arrivé dans la période des Fêtes de Noël. À ce moment-là je suis allé le chercher à l'aéroport, je l'ai amené à son appartement. Je l'ai accueilli du mieux que je pouvais. »(André ${ }^{11}$, informateur).

D'autres mettent à disposition des ressortissants français les services d'un avocat afin de s'occuper des démarches d'immigration nécessaires pour le professionnel au-delà de la procédure en lien avec l'Arrangement de reconnaissance mutuelle (ARM). Ceux qui ont pu disposer d'un avocat fourni par l'employeur pour s'occuper de leurs démarches d'immigration sont unanimes à en souligner les bénéfices. En effet, ils ont pu se concentrer davantage à la préparation de leur dossier pour intégrer l'Ordre en accord aux exigences de l'ARM.

Notons que ce type de pratiques est plus fréquent dans des firmes d'ingénierie faisant partie d'un groupe français ou bien ayant des filiales en France, familiers avec l'intégration de professionnels provenant de ce pays dans leur équipe.

\footnotetext{
${ }^{11}$ Tous les prénoms sont des pseudonymes.
} 
Nous avons également recueilli des témoignages informels d'une dizaine d'ingénieurs français lors d'un événement organisé par Francogénie ${ }^{12}$ le 24 février 2014, avec la présence de la responsable à l'admission de l'OIQ. Ces discussions ont souligné la présence d'entraves à l'entrée en pratique produites par la méconnaissance, chez certains employeurs, des exigences quant à la reconnaissance de diplômes dans le cadre de l'ARM. La multiplicité de plaintes à ce propos ainsi que l'importance accordée à cet aspect par l'OIQ ont abouti à la mise en place, par l'OIQ, de séances d'information concernant les ARM auprès de plusieurs employeurs. Ceux-ci prennent alors connaissance des règlements de l'ARM et de la non pertinence d'exiger une nouvelle reconnaissance du diplôme d'ingénieur, dans la mesure où celui-ci est reconnu par l'ARM. Cette méconnaissance de l'ARM parmi les employeurs a aussi été observée dans le cas des travailleurs sociaux et des architectes (Bédard et Roger, 2015).

«L'un de mes collègues, ayant son PRT ${ }^{13}$ délivré par l'OIQ, l'entreprise X lui a redemandé de faire les démarches officielles de reconnaissance de son diplôme ${ }^{14} \gg$. (Lö̈c, ingénieur français)

Le rôle de l'entreprise

Comme d'autres études sur l'intégration socioprofessionnelle des travailleurs immigrants qualifiés (Probyn, 1996; Syed, 2008; Valenta, 2008; Misiorowska, 2012; van Riemsdijk

\footnotetext{
${ }^{12}$ Pour ce qui est des ingénieurs français, «L'association québécoise Francogénie regroupe tous les diplômés de France et d'ailleurs issus des écoles d'ingénieurs et/ou d'universités offrant des formations scientifiques, dans le but de leur faciliter l'intégration professionnelle au Québec.»: http://francogenie.com/francogenie.

${ }^{13}$ Le «permis restrictif temporaire » permet à un candidat à l'admission à l'Ordre des ingénieurs du Québec (OIQ) dans le cadre de l'ARM France-Québec d'exercer sous la supervision d'un membre de l'OIQ, dans l'attente de la réussite des exigences d'admission.

${ }^{14}$ Être admissible à l'ARM implique que le diplôme et l'école française où l'ingénieur a fait ses études sont officiellement reconnus par l'OIQ.
} 
et al., 2016), nos analyses soulignent que l'entreprise devient l'un des acteurs centraux dans les processus d'apprentissage des codes professionnels et culturels parmi les professionnels formés à l'étranger. L'intégration au marché de l'emploi par le biais du transfert entre des filiales d'une même compagnie permet à l'ingénieur d'acquérir plus rapidement un poste à la hauteur de ses compétences, ou bien un poste avec des responsabilités similaires à celles assumées auparavant.

"C'est vrai que ça aurait été beaucoup plus difficile, surtout pour trouver, comme vous dites, un poste à la hauteur de mon expérience. Là en interne j'ai pu avoir finalement un poste à la hauteur de mes ambitions et de mon expérience et ne pas pâtir effectivement de la différence de poste qu'il aurait pu y avoir ou se créer. C'est vrai que quelque part je me considère un peu privilégié dans cette démarche; mais c'est pas tout le monde qui le fait, aussi bien, du jour au lendemain, 'je vais dans un nouveau pays et je m'intègre à zéro'. Ça aurait été compliqué de venir ici et de chercher du travail ici. Ça aurait été beaucoup plus compliqué effectivement. » (Thomas, ingénieur)

Les ingénieurs arrivés par un transfert intraentreprise disposent ainsi souvent de personnes-ressources les accompagnant dans leur processus de recherche d'un logement, l'accès aux services sociaux, au réseau scolaire, etc. Ceci permet d'accélérer les démarches non seulement pour l'entrée en pratique des professionnels français, mais aussi pour des aspects de la vie quotidienne. En ce sens, ceci facilite leur propre intégration socioculturelle ainsi que celle des membres de leurs familles. Ceci n'est pas spécifique aux Arrangements de reconnaissance mutuelle (ARM) et concerne plus largement la mobilité internationale via des entreprises multinationales. 
"Quand je suis arrivé, nous avons eu la chance d'avoir quelqu'un de mon entreprise qui s'occupe des Français qui arrivent. Il nous attendait déjà à l'aéroport... Il nous a aidés pour trouver un logement, m'expliquer qu'est-ce que je devais faire pour obtenir ma «carte-soleil »(assurance-maladie), inscrire mes enfants dans une garderie, aller chercher mon permis de conduire canadien, bref... ...tout ça nous a carrément aidés... au début on ne connaissait rien ! » (Victor, ingénieur)

Nouveau pays, nouveau contexte juridique et réglementaire...

L'existence des différences entre la France et le Québec quant à la réglementation de la profession d'ingénieur et au système universitaire qui régit la formation en génie pose des entraves à la compréhension des systèmes de chaque pays, et à l'intégration à l'Ordre des ingénieurs et l'exercice de la profession. Par exemple, en France, la Commission des titres d'ingénieurs (CTI) émet le titre d'ingénieur. Ce n'est donc pas un titre universitaire, tandis qu'au Québec, le titre d'ingénieur est émis suite à un diplôme universitaire. Également, les relevés de notes demandés par l'Ordre, documents couramment conservés suite aux études au Québec, sont difficiles à trouver pour un diplômé de la France, a fortiori pour ceux qui ont terminé leur formation il y a 20 ou 30 ans. Peu d'ingénieurs formés en France conservent une copie de leurs relevés de notes.

La production de preuves démontrant l'expérience professionnelle antérieure, requise par l'OIQ, n'est pas aisée non plus. Dans certains cas, la démarche est délicate, car ce faisant, le candidat dévoile son projet de départ à son employeur. Suite à ce constat, l'OIQ réfléchit aux exigences visant à documenter l'expérience professionnelle antérieure des ingénieurs, afin de tenir compte de ces difficultés. 
"La seule chose que je verrais qu'on pourrait moduler à l'occasion, nous on fonctionne avec des formulaires standards pour déclarer l'expérience professionnelle. Quelqu'un que son expérience « date», une attestation de travail, ou quoi que ce soit, n'est pas en mesure de remplir la documentation standard, à ce moment-là on pourrait avoir une mesure un peu compensatoire pour voir de quelle façon il pourrait nous démontrer son expérience. C'est la seule flexibilité qu'on aura pour justement démontrer de quelle façon l'expérience peut être présentée. » (André, informateur)

De plus, dans l'exercice de la profession d'ingénieur au Québec, la responsabilité professionnelle en lien avec la protection du public est portée par l'individu, tandis qu'en France, c'est l'entreprise qui juridiquement, assume la responsabilité de l'acte professionnel. L'ordre en France est plutôt une organisation de soutien à la profession. Au Québec, par le Code des professions, l'État délègue aux ordres professionnels la mission de protection du public, avec tous les processus disciplinaires qui en découlent. Selon l'OIQ, les exigences liées à la vérification des compétences des ingénieurs diplômés à l'étranger sont justifiées par cette mission qui lui incombe. Ceci surprend les professionnels formés à l'étranger, dont les ingénieurs français, parce que ce sont des spécificités juridiques découlant du Code des professions du Québec, qui structurent différemment l'exercice de la profession par rapport au contexte français.

"J'ai été surpris, car je pensais que l'OIQ était un organisme qui protégeait les ingénieurs. Et non, il est un organisme qui protège, plutôt, le public. (...) Il exerce un fort contrôle sur les ingénieurs. » (Luc, ingénieur)

Le discours du gouvernement québécois visant l'attraction des candidats à l'immigration 
Les ingénieurs rencontrés déplorent un discours gouvernemental visant à attirer les professionnels étrangers avec des informations ne correspondant pas avec l'expérience vécue d'intégration. Par exemple, des séances d'information sont organisées par le Ministère de l'Immigration, de la Diversité et de l'Inclusion du Québec (MIDI) en France pour attirer les candidatures d'immigrants qualifiés. Lors de ces séances, le discours d'agents gouvernementaux soulignerait la facilité d'accès à des places en garderie publique, des horaires de travail visant la conciliation travail-famille ou encore, la possibilité pour les conjoints de se trouver rapidement un emploi dans leur domaine.

«Il y a beaucoup, beaucoup de frustrations qui sont vécues par rapport à l'image que le Québec donne en France sur la terre d'accueil en facilitant les reconnaissances de compétences. Quand les gens arrivent ici, c'est pas toujours aussi facile et les gens voient le processus très long et ça crée énormément de difficultés pour eux-mêmes, pour l'immigrant qui vient travailler, et pour la conjointe ou le conjoint qui vient travailler, qui souvent laisse un métier en France, vient au Québec. On ne reconnaît pas le métier de cette personne-là, surtout dans le domaine de la santé. Ça, je dirais que ça crée énormément de frustrations. » (André, informateur)

Cet ingénieur témoigne de la difficulté à concilier le travail et la vie familiale.

«Par rapport à la conciliation famille et autre, pareil, je ne travaille pas moins qu'en France, j'ai moins de vacances. Oui, je travaille autant qu'en France, mais j’ai moins de vacances. Voilà. » (Victor, ingénieur)

Dans plusieurs cas, le projet de mobilité de ces ingénieurs répond à un projet familial, qui a donc des implications aussi pour les conjoints et les enfants, le cas échéant. Nos 
analyses relèvent également le lien entre la rétention et les conditions favorisant l'intégration sociale et professionnelle retrouvées par les ingénieurs et leurs proches. La possibilité de faire reconnaître les diplômés étrangers des conjoints et leur capacité à exercer la même profession qu'en France semblent des facteurs ayant un poids important dans la rétention.

Il existe des entraves à l'intégration socioprofessionnelle des conjoints/es dû au processus de reconnaissance des diplômes ainsi qu'à la difficulté d'obtenir des équivalences, qui entraîne d'autres démarches et des coûts afférents.

«On a fait aussi l'évaluation comparative des diplômes au MICC ${ }^{15}$. Là c'est pareil, il faut sortir un gros dossier papier, qu'il faut faire certifier conforme par un commissaire à l'assermentation, blablabla, avec les copies des diplômes, de l'identité, des relevés de notes par trimestre, par machin, des appréciations de stages, de trucs, de machins. Donc on fait un gros dossier. Ils ont pris un an à peu près pour étudier le dossier. (...) Donc ça a dî être un an ou un peu plus pour étudier le dossier, donc pour donner une espèce d'équivalence. Mais comme son métier n'est pas reconnu, bien, la formation ils l'ont pas reconnue non plus. » (Victor, ingénieur)

Les difficultés des conjoints à intégrer le marché de l'emploi représentent donc une entrave supplémentaire à l'intégration sociale.

"Le deuxième problème c'est que son métier français [celui de sa femme] n'est pas reconnu par aucun ordre ici. (...) un emploi qui lui aurait permis de

${ }^{15}$ Devenu depuis 2014, le MIDI (ministère de l'Immigration, de la Diversité et de l'Inclusion). 
s'intégrer encore plus facilement et tout ça. Parce qu'aujourd'hui, à date elle a rien trouvé. » (Victor, ingénieur)

Quant aux démarches concernant l'inscription à l'Ordre des ingénieurs du Québec, la plupart des professionnels interviewés soulignent l'importance de préparer le dossier à l'avance afin d'accélérer le processus une fois arrivés au Québec ${ }^{16}$. Plusieurs d'entre eux avaient commencé à préparer leur dossier un an à l'avance.

Cependant, les travailleurs français seraient, selon nos observations sur le terrain et les échanges avec des acteurs-clés, moins portés à recourir aux services d'aide à la recherche d'emploi que des immigrants d'autres origines.

«...la plupart des personnes qui assistent aux rencontres d'information ici, qui décident de suivre ce programme [programme de recherche d'emploi pour des nouveaux arrivants dans le domaine de l'ingénierie], sont surtout des diplômés provenant du Maghreb, deuxièmement des Africains, des Sud-Américains et en dernier lieu des Français. »(Jules, informateur)

Ceci semble associé au meilleur taux de placement des ingénieurs français, par rapport aux ingénieurs originaires d'autres pays, notamment ceux appartenant à des minorités visibles. De plus, notons que les ingénieurs formés en France disposent de groupes spécifiques, comme Francogénie.

"Le taux de placement des diplômés français est beaucoup plus élevé que celui du reste des étrangers. De plus, la plupart des ingénieurs diplômés en France

\footnotetext{
${ }^{16}$ Notons que l'OIQ a mis en place sur son site web «La Boussole». Cet outil permet aux ingénieurs étrangers d'avoir des renseignements utiles sur le cheminement et les étapes à franchir pour travailler comme ingénieur au Québec. http://www.oiq.qc.ca/fr/jeSuis/candidat/obtenirUnPermis/boussole/Pages/boussole.aspx
} 
qu'on a rencontrés cette dernière année ont eu un parcours assez réussi par rapport à leur intégration au marché de l'emploi. » (Alain, informateur)

La discrimination systémique entraine des barrières additionnelles reconnues par le système professionnel à l'admission et l'intégration professionnelle des immigrants, notamment des minorités visibles. C'est ce que reconnaît le Conseil interprofessionnel du Québec : « Même lorsqu'un candidat formé hors du Québec obtient son permis de l'ordre, l'insertion sur le marché du travail québécois peut être aussi semée d'embuches, particulièrement pour un professionnel issu d'une minorité visible, sans oublier l'importance de la maîtrise de la langue française »(Conseil interprofessionnel du Québec, $2016: 12)^{17}$.

\section{Le processus d'intégration socioprofessionnelle}

\section{b) dimension culturelle et relationnelle}

Si la dimension précédente nous permet de porter une analyse surtout au niveau macro, la dimension culturelle et relationnelle fait porter notre focus analytique au niveau micro. Nous examinons ici le rôle des individus dans leur expérience de recours aux structures mises en place pour l'intégration professionnelle et sociale.

De manière précise, cette deuxième dimension comprend: 1) le processus d'apprentissage des codes sociaux et culturels liés à l'emploi et à la vie sociale plus large, 2) les dynamiques relationnelles, personnelles et professionnelles ainsi que le rôle des réseaux sur l'expérience d'intégration à la société québécoise.

\footnotetext{
${ }^{17}$ De plus, nous avons constaté dans le cas d'un ingénieur d'origine africaine formé en France, un parcours d'insertion professionnelle plus difficile par rapport à ses collègues citoyens français. Ce témoignage a été recueilli en dépit du fait que ce professionnel ne se qualifiait pas pour l'admission à l'OIQ par le biais de l'Arrangement de reconnaissance mutuelle (ARM) France-Québec.
} 


\section{Analyse}

1) Le processus d'apprentissage des codes sociaux et culturels

Quant aux différences culturelles liées à l'emploi entre le Québec et la France, nos entrevues mettent en lumière un certain « choc culturel» vécu lors de l'intégration au marché de l'emploi québécois, ce qui rajouterait des difficultés à leur insertion autant professionnelle que personnelle. À ce propos, l'un de nos informateurs représentant une association avec une expertise avérée quant à ces situations ${ }^{18}$, abondait en ce sens :

«Mais ce que je peux vous dire c'est qu'au bout d'un an, on retrouve ces ingénieurs-là dans les cabinets de psychologues organisationnels parce qu'ils ont un problème d'attitude. D’attitude, pas de compétence (...) parce que leur comportement étant à la française, ça marche pas. Donc là ça marche pas avec leurs patrons, ça marche pas avec leurs employés, etc. » (Marc, informateur)

En ce sens, 1'Arrangement de reconnaissance mutuelle (ARM) n'aborderait pas ce qui est jugé comme le vrai défi des ingénieurs français émigrés au Québec : l'adaptation au nouvel environnement et à la culture du travail québécoise.

«Alors c'est pour ça que l'ARM, est-ce que ça élimine un obstacle qui était perçu comme celui qui empêchait l'intégration professionnelle? C'est faux. C'est pas là qu'est le défi. Le défi n'est pas au niveau de faire reconnaître son diplôme. Le défi est au niveau de s'adapter au nouvel environnement, s'adapter à la façon de faire ici. » (Marc, informateur)

\footnotetext{
${ }^{18}$ Le site internet de cette association mentionne qu'elle «regroupe tous les diplômés de France et d'ailleurs issus des écoles d'ingénieurs et/ou d'universités offrant des formations scientifiques, dans le but de leur faciliter l'intégration professionnelle au Québec ». Nous avons également pu constater l'importance et la vitalité de cette association.
} 
D'ailleurs, l’ARM France-Québec n’a pas été mis en place dans le but de faciliter l'intégration socioprofessionnelle. Il a été conçu avec des visées plus restreintes, i.e. faciliter l’admission à l’OIQ par les ingénieurs diplômés en France. Tout comme d'autres catégories de professionnels l'affirment (Bédard et Roger, 2015), la culture du travail au Québec s'apparente à ce qui est associé à l'Amérique du Nord : les rapports de pouvoir au sein des entreprises au Québec seraient moins hiérarchisés qu'en France. De plus, on souligne davantage les réalisations professionnelles plutôt que les caractéristiques des diplômes (nature de l'institution, du diplôme).

«Ici ils mettent à l'avance ton expertise et tes compétences tandis qu'en France les diplômes et l'école où tu as fait tes études c'est la première chose qu'on regarde. »(Denis, ingénieur)

Ces différences au niveau des cultures organisationnelles permettraient, en partie, de contrer les effets d'une déqualification professionnelle vécue durant les premières années d'entrée en pratique au Québec. Chez les ingénieurs, cette déqualification est vécue surtout durant la première année, lorsqu'ils disposent d'un permis restrictif temporaire (PRT). Ils doivent alors agir sous la supervision d'un ingénieur membre de l'OIQ, indépendamment des années d'expérience accumulées. L'ingénieur «junior» est parfois plus âgé et surtout, avec davantage d'expérience professionnelle, voire managériale que l'ingénieur québécois qui l'encadre. Surtout au début, cet encadrement peut être contraignant et donner l'impression d'une importante perte de temps dans l'insertion professionnelle dans l'entreprise. 
De plus, en France, le gain salarial est en lien avec le titre et le niveau d'études acquis par l'ingénieur alors qu'au Québec, le salaire est plutôt en lien avec la performance de l’ingénieur au sein de l'entreprise.

"... Ils disent, " je vais gagner plus en étant un master ". Moi je leur dis : non, je t'embauche comme ingénieur, pas comme master. Si tu me demandes plus cher, d'abord je vais te dire, tu commences comme ingénieur, selon les normes ici, les normes canadiennes, et si tu performes bien, c'est sûr que je vais te donner une augmentation, sur la base de ta performance, pas sur le fait que tu sois master» (Marc, informateur)

2) les dynamiques relationnelles personnelles et professionnelles ainsi que le rôle des réseaux

Nous avons mentionné plus haut que les ingénieurs français ont peu recours à des services communautaires de soutien à l'insertion professionnelle des immigrants qualifiés. Ils soulignent néanmoins l'efficacité de ces organismes, sur le plan de la connaissance de la société, de son fonctionnement, ainsi que pour la recherche d'emploi, puisqu'ils y ont recours pour l'insertion professionnelle de leur conjointe.

«Elle (ma femme) est allée voir les associations de quartier et autres, et notamment, à côté de là où on habitait, il y avait des ateliers, une sorte d'association communautaire où elle est allée, où elle a fait encore beaucoup de connaissances d'autres personnes. Donc très rapidement, oui elle a rencontré des gens qui sont devenus des amis. »(Denis, ingénieur) 
«D'ailleurs, ma femme était déjà rentrée en contact avec plusieurs associations et groupes ici à Montréal pour ne pas se retrouver toute seule et sans réseau. Ça nous a beaucoup aidés, car depuis le début nous avions un groupe d'amis. Bien que la plupart ce sont des immigrants comme nous. »(Luc, ingénieur)

«Les séminaires (dans les organismes communautaires) te permettent de rencontrer des gens et de commencer à avoir un réseau d'amis. Je le conseille à tout le monde. »(Jean, ingénieur)

Les différences culturelles apparaissent aussi à l'étape des techniques de recherche d'emploi. Tandis qu'en France la recherche d'emploi se fait surtout par l'envoi de CV, au Québec les techniques de réseautage (rencontres d'information, soirées de réseautage, réseaux sociaux sur internet, etc.) sont privilégiées (Béji et Pellerin, 2010). La proactivité individuelle des travailleurs devient essentielle à la réussite lors de la recherche d'emploi au Québec. À ce propos, les interviewés soulèvent l'efficacité des réseaux sociaux comme Linkedin dans la recherche d'emploi ainsi que des activités réseautage et des rencontres d'information avec les employeurs potentiels dans le cas du Québec.

"Ici au Québec [Linkedin] fonctionne très bien. Il est le meilleur moyen de rapidement établir un réseau professionnel et avoir des contacts ainsi que des offres d'emploi. » (Jean, ingénieur)

Finalement, les Français rencontrés constatent que le marché de l'emploi au Québec est beaucoup plus mobile et flexible qu'en France. Ceci expliquerait, en partie, que la mobilité des travailleurs au cours de leur carrière au Québec soit beaucoup mieux valorisée alors qu'en France, cette mobilité peut être signe d'incapacité à satisfaire les exigences, à s'intégrer à une entreprise au-delà du court terme, etc. 


\section{Conclusion}

Cette étude démontre l'importance d'une nouvelle logique de gestion des flux transnationaux de travailleurs qualifiés, dans ce cas-ci de professions réglementées, qui s'inscrit dans le paradigme de la mobilité. Celui-ci vise ici la mobilité de ces travailleurs dans le cadre d'une entente économique entre la France et le Québec et son éventuel élargissement vers un partenariat entre l’Union européenne et le Canada.

La perception initiale des ingénieurs français, quant au contexte de pratique et au système professionnel québécois, présume de l'existence de différences minimes par rapport à leur expérience française. Or, notre analyse suggère plutôt qu'en réalité, la pratique de la profession nécessite une adaptation importante des professionnels concernés. Ces différences peuvent se situer à divers niveaux : la formation universitaire, le contexte de pratique professionnelle, la régulation de celle-ci, de même que les pratiques de recherche d'emploi, l'évaluation en contexte professionnel, etc. Les processus mis en place par l'Ordre des ingénieurs du Québec (OIQ) pour l'obtention du permis reflètent ces considérations. Ils visent l'adaptation des professionnels français, formulés principalement dans le but de réaliser un aspect important de la raison d'être des ordres professionnels québécois : la protection du public.

L'étude illustre le fait que l'intégration socioprofessionnelle des ingénieurs éligibles à l'Arrangement de reconnaissance mutuelle France-Québec résulte d'une combinaison de facteurs relevant de l'ensemble des dimensions de l'intégration. Ainsi, la réussite socioprofessionnelle de ces travailleurs immigrants et par extension, leur rétention, s'explique par des facteurs structurels, socioculturels et relationnels. 
Comme d'autres études portant sur des accords de ce type l'ont soulevé (Gabriel, 2013), l'analyse du contenu et de la mise en place de l'ARM France-Québec pour les ingénieurs démontre que les accords internationaux de reconnaissance mutuelle facilitant la mobilité ne prévoient pas de mécanismes favorisant l'intégration sociale des travailleurs et de leurs proches à la société d'accueil. Ces types d'accords sont pensés uniquement en termes d'intégration au marché de l'emploi de l'individu-travailleur, en tenant seulement compte de la dimension économique des individus. L'aspect social et citoyen de leur intégration à la société d'accueil est passé sous silence. Pourtant, la mobilité internationale des professionnels a des implications au-delà de la sphère strictement économique et individuelle. Surtout, la rétention de ces professionnels passe par la capacité de ces derniers, et souvent de leurs proches, à intégrer le marché de l'emploi et à s'intégrer à la société d'accueil sous différentes facettes (logement, école, etc.). Ainsi, la rétention des professionnels dépendant de plusieurs autres facteurs que l'admission à l'ordre professionnel, il serait avantageux d'améliorer la gouvernance entre l'ensemble des acteurs concernés dans l'admission, l'intégration et la rétention de ces professionnels. Dans ce contexte de mobilité et d'internationalisation du travail, l'absence d'un régime international de régulation du marché de l'emploi laisse cette régulation aux gouvernements nationaux (Overbeek, 2002), ce qui implique une reconfiguration étatique avec de nouvelles formes de gouvernance et de nouveaux acteurs. Dans le cas qui nous occupe, soit celui du Québec, notons le rôle des ordres professionnels dans la gouvernance de l'admission des professionnels formés à l'étranger. Ces derniers ont à composer entre les intérêts de leur secteur d'activités, les pressions de sources publiques et gouvernementales à admettre des professionnels formés à l'étranger et leur mandat 
principal de protection du public. Ce faisant, ils visent un bénéfice à la fois pour les entreprises, les professionnels eux-mêmes et le public, et ce, autant sur le plan local qu'international. En effet, avant ces intérêts sectoriels et ceux du marché international, la protection du public s'impose comme mandat légal et réglementaire structurant les positions, décisions et pratiques des ordres professionnels. Dans cette configuration, la notion de protection du public prend une importance notoire. La résultante est la mise en place de mesures encourageant la mobilité internationale liée d'un côté, au marché de l'emploi et de l'autre, à la protection des intérêts locaux, essentiellement du public. Notons qu'inversement, des intérêts locaux et le discours médiatique plaident également en faveur de l'admission facilitée des professionnels formés à l'étranger. Les ordres professionnels sont souvent perçus dans le discours public comme étant à la défense de leurs membres. Justifiées par les ordres professionnels en vertu de la protection du public, les restrictions à l'admission des professionnels formés à l'étranger sont parfois perçues, dans le discours public, comme des contraintes abusives, les obligeant à accepter des emplois subalternes. Résumé rapidement, c'est le syndrome du professionnel devenant plutôt chauffeur de taxi.

Comme nous l'avons vu dans le cas des ingénieurs éligibles à l'ARM France-Québec, la protection du public en tant que notion juridique s'exprime par l'imposition de règles, responsabilités, obligations et limites à l'exercice de la profession qui semblent, aux yeux des nouveaux arrivants, contradictoires à l'esprit de ces accords, animés explicitement par l'idée d'une mobilité internationale.

Quant aux démarches d'admission à l'OIQ, plusieurs exigences semblent créer des entraves à l'entrée en pratique des professionnels visés par l'ARM dont notamment, la 
présentation des relevés de notes des études faites en France et la présentation des preuves concernant l'expérience professionnelle passée. L'OIQ a identifié ces dimensions et procède à la révision des mesures compensatoires exigées. En même temps, un nouveau débat s'ouvre au sein de l'OIQ sur l'évaluation par diplômes versus l'évaluation par compétences, ce qui en soi nécessiterait une analyse débordant du cadre de cet article. À propos de l'intégration socio-professionnelle des ingénieurs formés à l'étranger, nos analyses ${ }^{19}$ constatent l'absence de mesures prévues par l'Arrangement de reconnaissance mutuelle qui touchent aux dimensions culturelles et relationnelles de l'intégration socioprofessionnelle. Premièrement, cet instrument juridique ne prévoit pas de mesures facilitant l'intégration sociale et/ou professionnelle pour les accompagnants des professionnels éligibles (conjoint/es et enfants principalement). Ceci entraîne le découragement surtout des conjoint/es, pouvant mener à des remises en question du projet migratoire initial et de la décision de rester au Québec. Le projet professionnel de la plupart des professionnels interviewés n'est pas seulement un projet individuel, mais aussi familial. Les employeurs et les services mis en place par ceux-ci pour l'accueil et l'intégration des professionnels français à leur arrivée jouent un rôle très important dans le processus d'intégration de ces professionnels et de leurs proches, autant au marché de l'emploi que dans la société d'accueil. Les organismes d'aide à la recherche d'emploi ainsi que les organismes communautaires avec un mandat d'intégration sont également des ressources jugées intéressantes par les professionnels et leurs familles y ayant eu recours, autant pour la recherche d'emploi que pour la connaissance et l'intégration à la

\footnotetext{
${ }^{19}$ D'autres recherches sur les accords commerciaux et de mobilité internationale soulignent ce même constat (Nielson, 2002; Gabriel, 2013).
} 
société d'accueil. Ceci vaut même si ces organismes seraient moins fréquentés par les professionnels diplômés en France que les autres professionnels immigrant au Québec.

Deuxièmement, la logique sur laquelle ce type d'accord est bâti peut omettre les effets sur les professionnels visés du «choc culturel» que plusieurs d'entre eux peuvent expérimenter en raison des différences, souvent insoupçonnées, quant aux codes culturels liés à l'emploi et au monde du travail. Également, au moins au début, l'Arrangement de reconnaissance mutuelle ne prévoyait pas les impacts de la déqualification professionnelle - concrète et symbolique - des professionnels sous permis restrictif temporaire (PRT) limitant l'exercice de la profession. Toutefois, un marché de l'emploi beaucoup plus flexible et mobile au Québec qu'en France ainsi que l'existence de conditions salariales souvent meilleures, de dynamiques plus horizontales et de meilleures possibilités d'ascension en entreprises contribuent, en partie, à atténuer ces contraintes vécues par plusieurs ingénieurs.

En dépit de leur statut privilégié aussi bien sur les plans économique que social, les migrants qualifiés doivent apprendre les codes culturels de la société d'accueil et du milieu de travail de manière spécifique. Idéalement, le processus d'intégration socioprofessionnelle étant un processus à double sens - avec l'interaction entre les migrants et la société d'accueil (Béji et Pellerin, 2010), il serait pertinent d'approfondir nos analyses sur les acteurs non gouvernementaux ayant un rôle dans ce processus (employeurs, organismes communautaires d'aide à la recherche d'emploi, organismes représentant les intérêts des professionnels diplômés à l'étranger, etc.). Également, nous croyons pertinent d'élargir la présente recherche avec l'ajout de nouveaux cas, vu l'entrée 
en vigueur récente de l'ARM pour ingénieurs (juillet 2013) et ses ajustements subséquents.

Finalement, compte tenu des constats relevant de cette étude ainsi que d'études similaires portant sur l'intégration socioprofessionnelle des immigrants qualifiés, nous soulevons l'importance d'élargir notre étude à d'autres groupes professionnels de la même catégorie d'immigrants éligibles à l'ARM France-Québec. Ceci permettrait notamment de saisir ce qui, dans la démarche d'intégration socioprofessionnelle, est spécifique aux ingénieurs et ce qui traverse d'autres professions visées par des ARM. 


\section{RÉFÉRENCES}

Alba, Richard et Nee, Victor (1997) Rethinking assimilation theory for a new era of immigration, International Migration Review, 31 (4), pp. 826-874.

Arcand, Sébastien, Lenoir-Achdjian, Annick et Helly, Denise (2009) Insertion professionnelle d'immigrants récents et réseaux sociaux : le cas de Maghrébins à Montréal et Sherbrooke, Cahiers canadiens de sociologie, 34 (2), pp. 373-402.

Beaverstock, Jonathan V. (2011) Servicing British expatriate 'talent' in Singapore: Exploring ordinary transnationalism and the role of the 'expatriate' club, Journal of Ethnic and Migration Studies, 37(5), pp. 709-728.

--- (2005) Transnational elites in the city: British highly-skilled inter-company transferees in New York city's financial district, Journal of ethnic and migration studies, 31(2), pp. 245-268.

Bédard, Jean-Luc (2014) Mutual recognition arrangements between France and Quebec: Innovating on the transnational professional front, International Journal of Migration and Border Studies, 1 (1), pp. 127-134.

Bédard, Jean-Luc et Roger, Lucie (2015) Les parcours d'entrée à la pratique professionnelle de travailleurs français au Québec, dans le cadre des Arrangements de reconnaissance mutuelle France-Québec. Une analyse des facteurs facilitants et des contraintes auxquels font face huit catégories de professionnels, Montréal, INRS - Centre Urbanisation Culture Société, 102 p.

Béji, Kamel et Pellerin, Anaïs (2010) Intégration socioprofessionnelle des immigrants récents au Québec : le rôle de l'information et des réseaux sociaux, Relations industrielles/Industrial Relations, 65 (4), pp. 562-583. 
Bourdieu, Pierre (2000) Les structures sociales de l'économie, Paris, Seuil, coll. Liber, 289 p.

Chicha, Marie-Thérèse (2010) «Les immigrées universitaires et le marché du travail : discrimination systémique et intersectionnalité ». Présentation au $12^{\mathrm{e}}$ Congrès Metropolis, 19 mars, Montréal.

Cohen, Robin (2006) Migration and its enemies, Aldershot, Ashgate, 242 p.

Conseil interprofessionnel du Québec (2016) Mémoire sur le projet de loi 98 concernant l'admission aux professions réglementées et à la gouvernance du système professionnel, présenté à la Commission des institutions de l'Assemblée nationale du Québec, 23 août 2016).

Conseil interprofessionnel du Québec et Conférence des recteurs et des principaux des universités du Québec (2010) Accord de principe sur les mesures de compensation exigeant une formation universitaire dans le cadre d'un arrangement de reconnaissance mutuelle en vue de l'exercice d'une profession réglementée, [en ligne] consulté le 3 mars 2017. URL : http://www.crepuq.qc.ca/spip.php?article1320\&lang=fr.

Demers, Marc-André (2015) Frontières entre emploi, chômage et inactivité : la mesure du chômage a-t-elle omis plus de 280000 personnes en 2014?, Flash-info travail et rémunération, 16(2), pp.1-17. Institut de la statistique du Québec. [En ligne] consulté le 18 décembre 2017. URL: http://www.stat.gouv.qc.ca/statistiques/travail$\underline{\text { remuneration/bulletins/flash-info-201506.pdf }}$

Dumas-Martin, Antoine (2014) L'Entente Québec-France et ses limites : le cas des médecins de famille, [en ligne] consulté le 12 mars 2017. URL: http://hdl.handle.net/11143/5445 
Faulconbridge, James R., Beaverstock, Jonathan V., Derudder, Ben, et al. (2009) Corporate ecologies of business travel in professional service firms: Working towards a research agenda, European Urban and Regional Studies, 16(3), pp. 295-308.

Favell, Adrian, Miriam Feldblum et Michael P. Smith (2007) The human face of global mobility: a research agenda, Society, 44 (2), pp. 15-25.

Findlay, Allan M., Li, F. Lin N., Jowett, A. John, et al (1996) Skilled International Migration and The Global City: A Study of Expatriates in Hong Kong, Transactions of the Institute of British Geographers, 21, pp. 49-61.

Friesen, M. R. (2011) Immigrants' integration and career development in the professional engineering workplace in the context of social and cultural capital, Engineering Studies, 3 (2), pp. 79-100.

Gabriel, Christina (2013) North American Free Trade Agreement (NAFTA), Skilled Migration, and Continental Nursing Markets, Population, Space and Place, 19 (4), pp. 389-403.

Gauthier, Carol-Ann (2013) Le rôle des réseaux sociaux dans le processus d'intégration socioprofessionnelle des femmes immigrantes qualifiées au Québec, Économie et solidarités, 43 (1-2), pp. 98-110.

Gordon, Milton A. (1978) Human Nature, Class and Ethnicity. New York, Oxford University Press, $316 \mathrm{p}$.

Hawthorne, Lesleyanne (2015) The impact of skilled migration on foreign qualification recognition reform in Australia, Analyse de politiques, 41 (Supplement 1), pp. S173-S187. 
--- (2013) Recognizing foreign qualifications. Emerging global trends, Washington, D.C., Migration Policy Institute, 17 p.

Heckmann, Friedrich (2005) Integration and Integration Policies, [en ligne] European Forum for Migration Studies, Bamberg, consulté le 20 février 2016. URL: http://www.efms.uni-bamberg.de/pdf/INTPOL\%20Final\%20Paper.pdf

Heckmann, Friedrich et Dominique Schnapper (eds.) (2003) The Integration of Immigrants in European Societies. Stuttgart, Lucius and Lucius, $261 \mathrm{p}$.

Houle, France (2015) Regard sur l'Entente Québec-France et ses Arrangements de reconnaissance mutuelle, Vie économique, 7 (1), pp. 1-7.

Kofman, E. (2005) Figures of the cosmopolitan: privileged nationals and national outsiders. Innovation, European Journal of Social Science Research, 18 (1), pp. 83-97.

Lahire, Bernard (2012) Monde pluriel. Penser l'unité des sciences sociales, Paris, Seuil, coll. La couleur des idées, 393 p.

Larbiou, Benoît (2008) L'immigration organisée. Construction et inflexions d'une matrice de politique publique (1910-1930) [en ligne], Asylon(s), (4), consulté le 17 février 2016. URL : http://www.reseau-terra.eu/article735.html

--- (2003). Connaître et traiter l'étranger: Les constructions sociales d'un savoir politique sur l'immigration 1914-1945, Thèse de doctorat, Université Montpellier 1, 880 p.

Lin, Nan (1999) Social Networks and Status Attainment, Annual Review of Sociology, 25 , pp. $467-487$. 
Lockwood, David (1964) "Social Integration and System Integration" dans Zollschan, K. et Hirsch, Walter (eds.), Explorations in Social Change. London, Routledge and Kegan.

Mendoza, Dovelyn Rannveig, Demetrios G. Papademetriou, Maria Vincenza Desiderio, Brian Salant, Kate Hooper, K. et Taylor Elwood (2017) Reinventing Mutual Recognition Arrangements: Lessons from International Experiences and Insights for the ASEAN Region. Asian Development Bank.

Misiorowska, Mariola (2012). Les trajectoires socioprofessionnelles des nouveaux arrivants, travailleurs qualifiés au Québec. Qu'est-ce qu'une «intégration réussie»? Thèse de doctorat. Université de Montréal (Canada).

Mercier, Carole (2012), The Québec-France Agreement on the Mutual Recognition of Professional Qualifications, Journal of Nursing Regulation, 2 (4), pp. 53-57.

Meyer, Jean-Baptiste, David Kaplan et Jorge Charum (2001) Nomadisme des scientifiques et nouvelle géopolitique du savoir, Revue Internationale des Sciences Sociales, 168 (2), pp. 341-354.

Millar, John et Jane Salt (2008) Portfolios of Mobility: The Movement of Expertise in Transnational Corporations in Two Sectors - Aerospace and Extractive Industries, Global Networks, 8 (1), pp. 25-50.

Ministère des relations internationales et de la Francophonie (MRIF) (2011) Entente entre le Québec et la France en matière de reconnaissance mutuelle des qualifications professionnelles, [en ligne] consulté le 28 juillet 2017. URL: http://www.mrif.gouv.qc.ca/Content/documents/fr/2008-12.pdf 
Myrdal, Gunnar (1944) An American dilemma, Volume 2: The Negro problem and modern democracy (Vol. 2). Transaction Publishers.

Nedelcu, Mihaela (2005) Stratégie de migrations et d'accès au marché du travail des professionnelles roumaines à Toronto, Revue européenne des migrations internationales, 21 (1), pp. 77-106.

Nielson, Julia (2002) Current Regimes for Temporary Movement of Service Providers [en ligne], communication présentée au Symposium conjoint OMC - Banque Mondiale : Movement of Natural Persons under the GATS, Genève, consulté le 10 avril 2016. URL : http://www.wto.org

Noiriel, Gérard (2008) L'immigration : naissance d'un «problème » (1881-1883) [en ligne], Revue Agone, 40, pp. 15-40, consulté le 6 avril 2016. URL: http://agone.org/revueagone/agone40/enligne/1/.

--- (2001) État, nation et immigration : vers une histoire du pouvoir, Paris, Belin, 399

p.

Ordre des ingénieurs du Québec (OIQ) (2017a) Information générale [en ligne], consulté le 20 décembre 2017. URL : https://www.oiq.qc.ca/fr/jeSuis/membre/juniorat/examenProfessionnel/Pages/Information $\stackrel{\text { aspx }}{a}$

Ordre des ingénieurs du Québec (OIQ) (2017b) Évaluation du dossier. Diplôme d'ingénieur obtenu en France, [en ligne] consulté le 20 décembre 2017. URL :https://www.oiq.qc.ca/fr/jeSuis/candidat/obtenirUnPermis/diplomeIngenieurFrance/Pages levaluationDossier.aspx. 
Organisation Internationale pour les Migrations (2008) Rapport 2008 sur l'état de la migration dans le monde [en ligne], consulté le 18 janvier 2016. URL :

http://www.iom.int/jahia/Jahia/newsArticleEU/cache/offonce/lang/fr?entryId=20343.

Overbeek, Henk W. (dir.) (2002) Restructuring Hegemony in The Global Political Economy: The Rise of Transnational Neo-liberalism in the 1980s, Londres, Routledge, 293 p.

Papademetriou, Demetrios G., Sumption, Madeleine et Somerville, Will (2009) Migration and the Economic Downturn: What to expect in the European Union, Washington, D.C., Migration Policy Institute, 15 p.

Pellerin, Hélène (2011) De la migration à la mobilité : changement de paradigme dans la gestion migratoire. Le cas du Canada, Revue Européenne des Migrations Internationales, 27 (2), pp. 57-75.

Portes, Alejandro et József Böröcz (1989) Contemporary Immigration: Theoretical Perspectives on its Determinants and Modes of Incorporation, International Migration Review, 23 (3), pp.606-630.

Probyn, Elspeth (1996) Outside Belongings, Londres, Routledge, 181 p.

Rawls, John (1999 [1971]) A Theory of Justice. Harvard, Harvard University Press, 607 p.

Rex, John and Tomlinson, Sarah (1979) Colonial Immigrants in a British city. A Class Analysis. London, Routledge.

Syed, Jawad (2008) Employment Prospects for Skilled Migrants: A Relational Perspective, Human Resource Management Review, 18 (1), pp. 28-45. 
Valenta, Marko (2008) The Workplace as an Arena for Identity Affirmation and Social Integration of Immigrants, [en ligne] Forum Qualitative Social Research, 9 (2), consulté le 6 novembre 2015. URL : http://nbn-resolving.de/urn:nbn:de:0114-fqs0802140 van Riemsdijk, Micheline, Scott Basford et Burnham, Alana (2016) Socio-Cultural Incorporation of Skilled Migrants at Work: Employer and Migrant Perspectives, International Migration, 54 (3), pp. 20-34.

Walsh, Katie (2006) Dad says I'm tied to a shooting star! Grounding (research on) British expatriate belonging, Area, 38 (3), pp. 268-278.

Weygold, Serge A., Alain Berset, Olivier Crevoisier et François Hainard (2001) La politique d'admission de la main-d'oeuvre étrangère : désirabilité, acceptabilité, intégrabilité, Revue suisse de sociologie, 27 (1), pp. 117-129. 